\title{
Radiosensitization of non-small cell lung cancer by kaempferol
}

\author{
WEI-TING KUO ${ }^{1}$, YUAN-CHUNG TSAI ${ }^{2}$, HIS-CHIN WU ${ }^{3}$, YUNG-JEN HO ${ }^{4}$, \\ YUEH-SHENG CHEN ${ }^{4,5}$, CHEN-HAN YAO ${ }^{6}$ and CHUN-HSU YAO ${ }^{4,5,7}$ \\ ${ }^{1}$ Institute of Biomedical Engineering, National Taiwan University, Taipei $10051 ;{ }^{2}$ Graduate Institute of Clinical \\ Medical Science; ${ }^{3}$ School of Medicine; ${ }^{4}$ Department of Biomedical Imaging and Radiological Science; \\ ${ }^{5}$ School of Chinese Medicine, China Medical University; ${ }^{6}$ Viator Catholic High School, Taichung 40402; \\ ${ }^{7}$ Department of Biomedical Informatics, Asia University, Taichung 41354, Taiwan, R.O.C.
}

Received June 3, 2015; Accepted June 24, 2015

DOI: $10.3892 / o r .2015 .4204$

\begin{abstract}
The aim of the present study was to determine whether kaempferol has a radiosensitization potential for lung cancer in vitro and in vivo. The in vitro radio-sensitization activity of kaempferol was elucidated in A-549 lung cancer cells by using an MTT (3-(4 5-dimethylthiazol-2-yl)-25-diphenyltetrazolium bromide) assay, cell cycle analysis and clonogenic assay. The in vivo activity was evaluated in the BALB/c nude mouse xenograft model of A-549 cells by hematoxylin and eosin staining and immunohistochemistry, and the tumor volume was recorded. Protein levels of the apoptotic pathway were detected by western blot analysis. Treatment with kaempferol inhibited the growth of A-549 cells through activation of apoptotic pathway. However, the same doses did not affect HFL1 normal lung cell growth. Kaempferol induced G2/M cell cycle arrest and the enhancement of radiation-induced death and clonogenic survival inhibition. The in vivo data showed that kaempferol increased tumor cell apoptosis and killing of radiation. In conclusion, the findings demonstrated that kaempferol increased tumor cell killing by radiation in vitro and in vivo through inhibition of the AKT/PI3K and ERK pathways and activation of the mitochondria apoptosis pathway. The results of the present study provided solid evidence that kaempferol is a safe and potential radiosensitizer.
\end{abstract}

\section{Introduction}

Lung cancer is the leading cause of cancer-associated mortalities worldwide, with an estimated 1.61 million new cases and 1.38 million mortailies in $2008(1,2)$. Approximately $85 \%$ of

Correspondence to: Dr Chun-Hsu Yao, Department of Biomedical Imaging and Radiological Science, China Medical University, 91 Hsueh-Shih Road, Taichung 40402, Taiwan, R.O.C.

E-mail: chyao@mail.cmu.edu.tw

Key words: kaempferol, radiosensitizer, lung cancer, radiation, apoptosis primary lung cancer patients have non-small-cell lung cancer (NSCLC) (3). Surgery is the standard treatment for early-stage NSCLC. However, the majority of patients diagnosed at an advanced stage are unsuitable for surgical resection or extensive mediastinal lymphadenopathy $(4,5)$. Chemo- and radiotherapy are the current standard of care for patients with unresectable advanced NSCLC $(6,7)$. However, there are several limiting factors of chemo- and radiotherapy, including dose tolerance limitation of normal tissue and tumor radioresistance (8). Accordingly, identifying effective agents for enhancing tumor sensitivity to radiation and reducing adverse effects on normal tissues are crucial.

Acting as radiosensitizers, drugs can accelerate the killing of cancer cells by increasing the effectiveness of radiation with little effect on normal cells (9). The PI3K/Akt and ERK pathways play an essential role in confirming sensitivity or resistance to radiation by inhibiting a survival pathway that induces cell apoptosis $(10,11)$. Previous studies also showed that treatment with the ERK inhibitor can reduce radioresistance and suppressed activation of PI3K/Akt can promote radiosentization $(12,13)$. Inhibition of growth of cancer cells and the induction of apoptosis are important determinants of the response to anticancer therapy (14).

Bioflavonoids, which are the phytochemicals that are abundant in a variety of plants have a vital role in cancer prevention as they can scavenge free radicals $(15,16)$. Kaempferol is a flavonol that is present in tea, broccoli, grapefruit, Brussels sprouts and apples. It is claimed to have an anti-proliferative effect on colon cancer cell lines $(17,18)$. The anti-angiogenic properties of kaempferol have also been well documented (19). Among the flavonols, kaempferol is absorbed particularly well when administered orally, even in low doses, with minimal inter-individual variation (20). Kaempferol is reportedly effective against pancreatic cancer, human lung non-small carcinoma and glioma cells (21-23). It has been reported to act synergistically with quercetin to cause a considerable anti-proliferative effect in human gut cells and breast cancer cells (24). However, the combination of kaempferol with radiation against cancer remains to be evaluated. In the present study, the anticancer capacity of kaempferol and its ability to sensitize tumors radiation were assessed in in vitro and in vivo 
studies. Its effects on signal transduction in lung cancer cells were also investigated.

\section{Materials and methods}

Materials and chemicals. Kaempferol was purchased from Sigma-Aldrich (St. Louis, MO, USA). All other chemicals and reagents were of analytical grade. Chemicals were obtained from Sigma-Aldrich, except where otherwise indicated. The antibodies for $\alpha$-tubulin, p-ERK (E-4) and PI3K $\mathrm{p} 85 \alpha(\mathrm{Z}-8)$ were purchased from Santa Cruz Biotechnology, Inc. (Santa Cruz, CA, USA). The antibodies for phospho-Akt (Ser473) and caspase-7 were purchased from Cell Signaling Technology (Danvers, MA, USA). The antibody for caspase-3 was purchased from Millipore (Billerica, MA, USA). MTT (3-(4,5-dimethylthiazol-2-yl)-2,5-diphenyltetrazolium bromide) was purchased from USB Corp. (Cleveland, $\mathrm{OH}$, USA). The Annexin V conjugates and PI (propidium iodide) solid were all purchased from Invitrogen Corp. (Carlsbad, CA, USA).

Cell culture. The human A-549 lung carcinoma and the human HFL1 normal lung fibroblast cell lines were obtained from BCRC (Bioresource Collection and Research Center) in Taiwan. A-549 cells were cultured in Ham's F12K medium supplemented with $10 \%$ fetal bovine serum (FBS), $2 \mathrm{mM}$ L-glutamine and $1.5 \mathrm{~g} / 1$ sodium bicarbonate. HFL1 cells were also cultured in Ham's F12K medium supplemented with $10 \%$ FBS. The cells were maintained under standard cell culture conditions at $37^{\circ} \mathrm{C}$ and $5 \% \mathrm{CO}_{2}$ in a humid environment. Adherent cells were harvested using trypsin and re-suspended in a serum-containing medium before use in the assays, as described below.

MTT assay. MTT assay was performed to determine the effect of kaempferol on cell growth. This assay is based on the cleavage of the yellow tetrazolium salt MTT to the purple formazan crystal by mitochondrial succinate dehydrogenase from living cells. This reduction occurs only when mitochondrial reductase enzymes are active, thus the extent of the reduction is directly associated with the number of viable cells.

Briefly, A-549 cells were seeded at a density of $1 \times 10^{4}$ cells/well in 24-well plates and pre-incubated for $24 \mathrm{~h}$. The medium was then replaced with serum-free medium, to which indicated doses of kaempferol were added for $48 \mathrm{~h}$. Following treatment with kaempferol, the medium was replaced with $0.5 \mathrm{mg} / \mathrm{ml}$ MTT medium and incubated for $4 \mathrm{~h}$. The MTT solution was removed from the wells and the formazan crystals were dissolved in DMSO. The concentration was then determined using a microplate reader at $570 \mathrm{~nm}$.

Western blot analysis. After the cells were treated with kaempferol, they were rinsed three times with PBS. The cells were then directly solubilized on ice in a lysis buffer $(0.5 \mathrm{M}$ Tris- $\mathrm{HCl}$, $\mathrm{pH} 7.4,1.5 \mathrm{M} \mathrm{NaCl}, 2.5 \%$ deoxycholic acid, 10\% NP-40, $10 \mathrm{mM}$ EDTA) that contained a protease inhibitor cocktail. After $5 \mathrm{~min}$, the cells were scraped and the lysate was collected in an Eppendorf tube. The lysate was cleared by centrifugation at $12,000 \mathrm{x} \mathrm{g}$ for $30 \mathrm{~min}$ at $4^{\circ} \mathrm{C}$, and the protein concentration in the supernatant was determined by the Bradford method (BioRad protein assay; Hercules, CA, USA).

For western blotting, equal amounts of proteins were resolved over $10-12 \%$ sodium dodecyl sulfate polyacrylamide gel electrophoresis (SDS-PAGE) and transferred onto a polyvinylidene fluoride (PVDF) membrane. The non-specific sites on the blots were blocked by incubating them in a blocking buffer (5\% non-fat dry milk/TBS, $\mathrm{pH} 7.4$ ) for $1 \mathrm{~h}$ at room temperature. Incubation was performed using an appropriate monoclonal primary antibody in TBS overnight at $4^{\circ} \mathrm{C}$, and then incubated with a horseradish peroxidase-conjugated secondary antibody for $1 \mathrm{~h}$ at room temperature. Immunoreactive bands were visualized using an enhanced chemiluminescence system.

Cell cycle analysis. To determine the effect of kaempferol

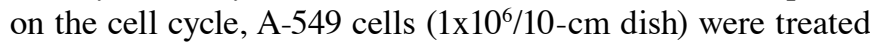
with $56 \mu \mathrm{M}$ kaempferol for the indicated time points $(0,3,6$, 12, 24 and 48 h). Each time-point was evaluated with at least three plates. Briefly, the cells were treated with kaempferol at the specified time points. The cell pellet was washed with cold phosphate-buffered saline (PBS), fixed with $95 \%$ ethanol overnight, and stained with $1.0 \mathrm{ml} \mathrm{PI} /$ Triton X-100 for $30 \mathrm{~min}$ in the dark. The stained cells were analyzed using a flow cytometer (BD FACSCanto; BD Biosciences, San Diego, CA, USA). The data were analyzed using ModFit LT 3.0 software (Verity Software House, Inc., Topsham, ME, USA).

Radiation exposure. The A-549 cells in dishes with diameters of $10 \mathrm{~cm}$ were exposed to radiation by photons from a linear accelerator. The dishes were placed within the exposure area on an acrylic sheet with an area of $25 \times 25 \mathrm{~cm}$ and covered by a bolus with an area of $1.5 \times 1.5 \mathrm{~cm}$. The cells were exposed to the indicated doses $(2,4,6,8,10$ and 12 Gy). The summarized radiation dosage on the cells and exposure period was well calculated and monitored from the control room. Following exposure, each dish was immediately transferred to a cell culture incubator.

Clonogenic assay. Cell survival via radiation alone or the combination of kaempferol with radiation was analyzed using a clonogenic assay. A-549 cells were seeded in 10-cm dishes with an appropriate amount of radiation or kaempferol with radiation. The cells were exposed to $0,2,4,6,8,10$ and 12 Gy radiation and treated with $56 \mu \mathrm{M}$ kaempferol for $48 \mathrm{~h}$, followed by different doses of radiation. The cell medium was replaced with a new medium and cultured in an incubator for two weeks. After fixation and staining with $5 \%$ crystal violet, colonies containing $\geq 50$ cells were counted under a microscope. The plating efficiency was defined as the average number of cell colonies counted divided by the number of initial cells for the control group which were not exposed to kaempferol or radiation. The surviving fraction was determined as the average number of cell colonies counted divided by the number of seeding cells multiplied for the plating efficiency.

Tumor xenograft studies. A-549 cells $\left(2 \times 10^{6}\right.$ in $\left.200 \mu 1 \mathrm{PBS}\right)$ were implanted subcutaneously in BALB/c nude mice near the left hind leg. The mice were obtained from BioLasco Taiwan Co., Ltd. (Taipei, Taiwan). The tumors were allowed to reach a volume of $350 \mathrm{~mm}^{3}$ prior to initiation of treatment (3 weeks 
after tumor implantation). The mice ( 3 mice per group) were treated (intraperitoneally) with radiation at 4 Gy alone or radiation plus kaempferol ( $4 \mathrm{~h}$ before radiation). The tumor volume and size were recorded every 5 days.

Histological stains. The mice were sacrificed and tissues were harvested and placed in tissue wells filled with Tissue-Tek OCT. The tissue wells were rapidly frozen and then dried. The frozen specimens were sectioned using a cryostat microtome. hematoxylin and eosin (H\&E)-stained specimens were used to determine histological morphology and nuclear structures. An anti-caspase-3 immunohistochemical (IHC) stain was used to label the apoptotic cells. The cryosections were fixed with $4 \%$ formaldehyde overnight and washed with PBS. Non-specific binding sites were blocked with $2 \%$ (w/v) BSA solution in PBS prior to labeling with antibody. The specimens were washed several times with PBS, and incubated overnight at $4^{\circ} \mathrm{C}$ with a 1:200 dilution of polyclonal anti-caspase-3 antibody. The sections were then counterstained with hematoxylin and mounted on a cover slide for optical microscopic examination.

Statistical analysis. Numerical data were presented as mean \pm standard deviation from at least three experiments. Statistical comparisons were made using the Student's t-test or one-way analysis of variance (ANOVA) followed by post hoc Fisher's LSD multiple comparison test, as indicated. Any difference was regarded as significant at a probability of $\mathrm{P}<0.05$. Data were analyzed using the SPSS software version 10.0 (SPSS, Inc., Chicago, IL, USA).

\section{Results}

Kaempferol selectively suppresses the growth of A-549 cells and induces cell apoptosis through inhibition of PI3K/AKT and ERK pathways. The cell viability of A-549 lung cancer cells and HFL1 normal lung cells when treated with kaempferol was assessed using an MTT assay. As shown in Fig. 1A, the treatment of A-549 cells with kaempferol at doses of $14-112 \mu \mathrm{M}$ inhibited growth by $28,57,65$ and $68 \%$. The same doses did not inhibit the growth of HFL1 normal lung cells (Fig. 1A). The inhibitory effects on the two cell lines differed significantly $(\mathrm{P}<0.05)$.

To elucidate the mechanism that underlies the kaempferolinduced death of A-549 cells, the roles of caspases were investigated by examining their activation. As shown in Fig. 1B, the expression of caspase-7 increased significantly with the dose of kaempferol. Since members of the Bcl-2 family are the main regulators of apoptosis, the effect of kaempferol on the levels of such proteins in A-549 cells was determined. Western blot analysis revealed a dose-dependent increase in the expression of Bax, verifying the induction of the apoptotic process. Inhibition of the PI3K/AKT and ERK pathways also increased the level of Bax, thus, we also detected the phosphorylation of AKT, PI3K and ERK. Additionally, kaempferol suppressed the phosphorylation of AKT, PI3K and ERK.

Kaempferol induces G2/M cell cycle arrest. To determine whether kaempferol was a radiosensitizer, cell cycle analysis was first performed. The effects of kaempferol on the cell cycle progression of A-549 cells are shown in Fig. 2. There
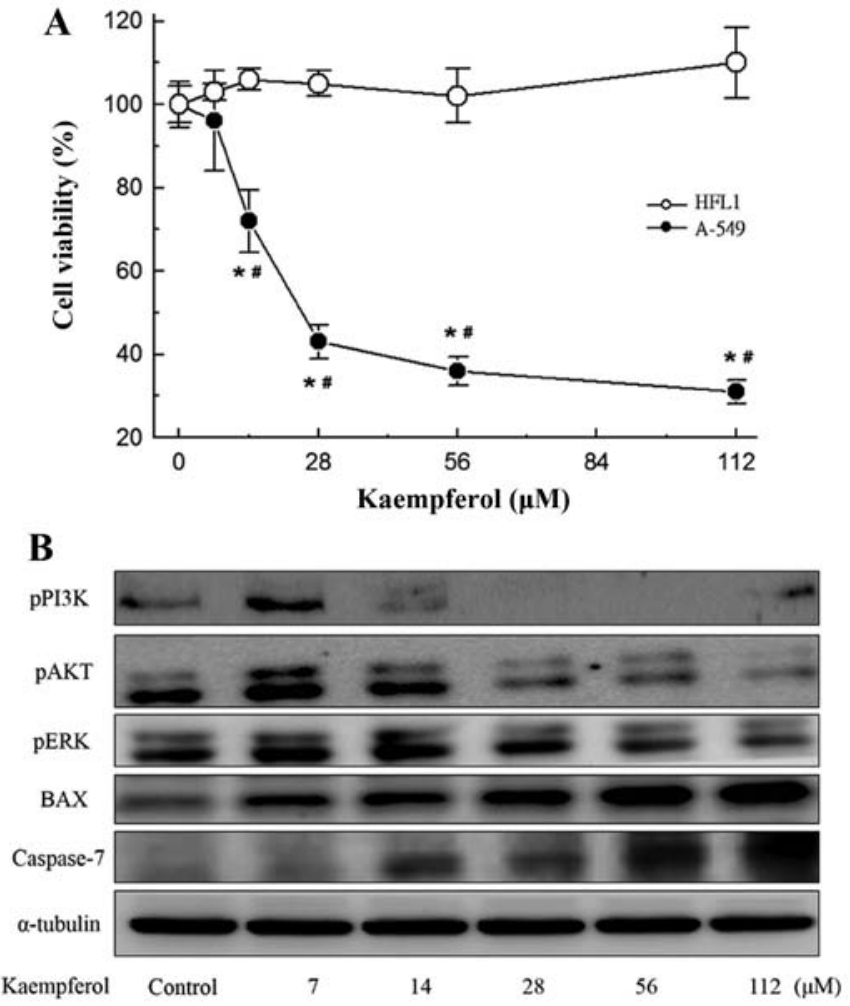

Figure 1. Effects of kaempferol on cell viability and protein expression. (A) Treatment with kaempferol (7-112 $\mu \mathrm{M})$ on A-549 and HFL1 cells for $48 \mathrm{~h}$. The data shown are mean \pm SD for $\mathrm{n}=6 .{ }^{*} \mathrm{P}<0.05$ vs. control; ${ }^{\#} \mathrm{P}<0.05$ vs. counterpart treatment. (B) pPI3K, pAKT, pERK, Bax and caspase-7 expression of A-549 cells.

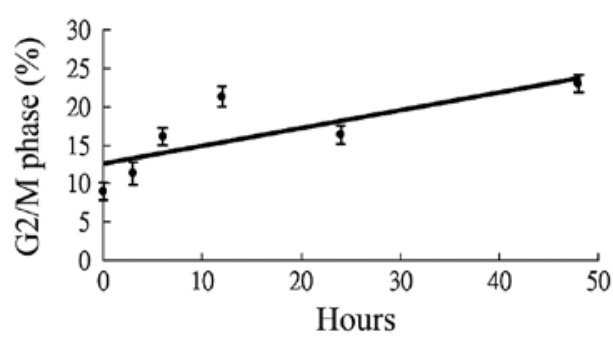

Figure 2. Cell cycle of A-549 cells after treatment with kaempferol $(56 \mu \mathrm{M})$.

was an increase in the cell population in the $\mathrm{G} 2 / \mathrm{M}$ phase at $48 \mathrm{~h}$ compared with the other groups $(0,3,6,12$ and $24 \mathrm{~h})$ following treatment with $56 \mu \mathrm{M}$ of kaempferol. The trend line of the $\mathrm{G} 2 / \mathrm{M}$ phase showed that the cell population exhibited a time-dependent increase following treatment.

Kaempferol induces enhancement of radiation-induced death and retards phosphorylation of PI3K and ERK. Kaempferol at a dose of $14 \mu \mathrm{M}$, which inhibited cell growth by $28 \%$ (Fig. 1A), was used to examine the radiosensitizing effects of kaempferol on A-549 cells. As shown in Fig. 3A, radiation exposure alone inhibited cell growth dose-dependently from 2 to $12 \mathrm{~Gy}$. Kaempferol treatment at $48 \mathrm{~h}$ prior to radiation exposure enhanced inhibition of cancer cell growth from 2 to 6 Gy. The application of kaempferol prior to radiation exposure enhanced A-549 cell radiosensitivity and strengthened the inhibitory effects. 


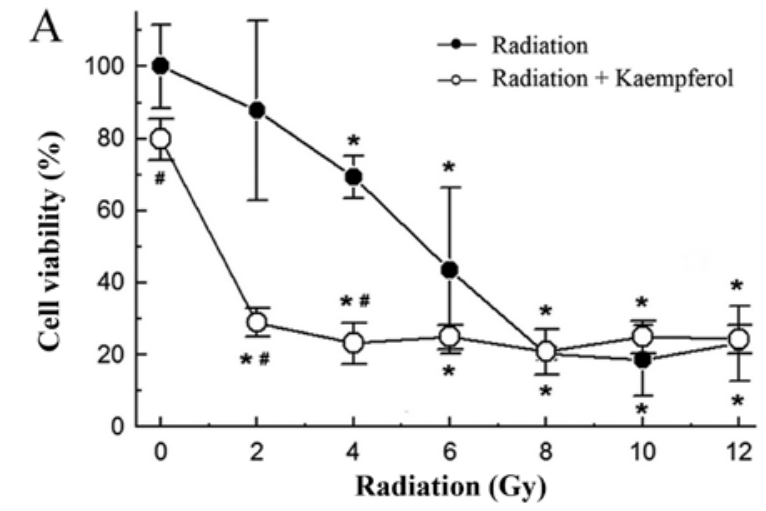

B

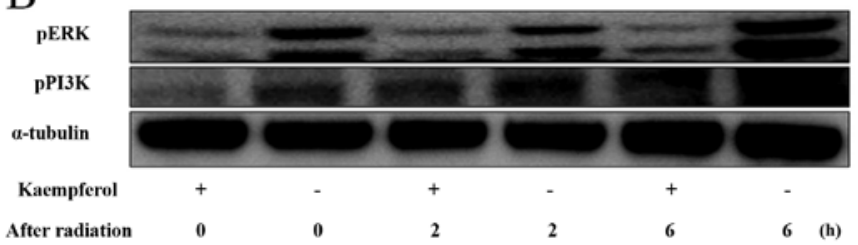

Figure 3. Effects of radiation only and radiation plus kaempferol $(14 \mu \mathrm{M})$ on cell viability and protein expression of A-549 cells. (A) Treatment with kaempferol for $48 \mathrm{~h}$ before radiation exposure and radiation only. The data shown are mean $\pm \mathrm{SD}$ for $\mathrm{n}=6$. ${ }^{*} \mathrm{P}<0.05$ vs. control; ${ }^{\# \mathrm{P}}<0.05$ vs. counterpart treatment. (B) pPI3K and pERK expression following treatment with radiation only and plus kaempferol before radiation exposure.

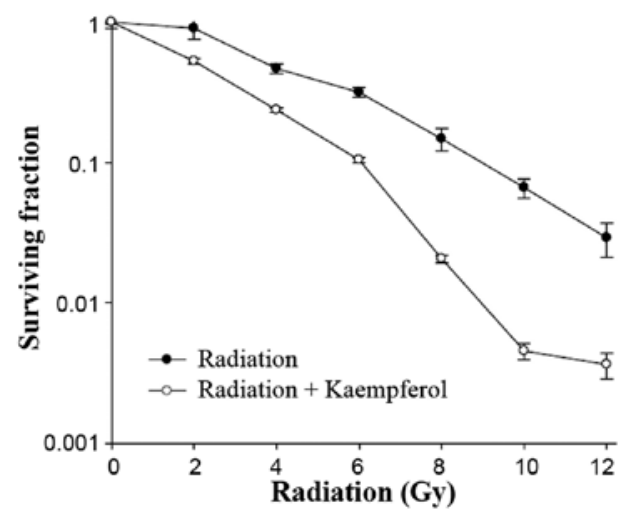

Figure 4. Effect of radiation only and radiation plus kaempferol on surviving fraction by clonogenic assays. Treatment with kaempferol $(56 \mu \mathrm{M})$ for $48 \mathrm{~h}$ before radiation exposure and radiation only on A-549 cells.

To achieve a good prognosis, inhibition of radioresistant protein PI3K or ERK activated after radiation exposure was crucial. We found treatment with kaempferol before radiation exposure inhibited PI3K and ERK, and reduced phosphorylation of ERK sustainability after radiation exposure of $6 \mathrm{~h}$ (Fig. 3B).

Kaempferol decreases the clonogenic survival. To determine the effects of kaempferol on the radiosensitivity of A-549 cells, clonogenic assays were performed after exposure to $0-12 \mathrm{~Gy}$ of radiation with and without 48 -h pretreatment with $56 \mu \mathrm{M}$ of kaempferol. We found that treatment of the cells with radiation alone led to a minimal effect on clonogenic survival. However, when pretreated with kaempferol before radiation exposure, the surviving fraction decreased significantly (Fig. 4). The

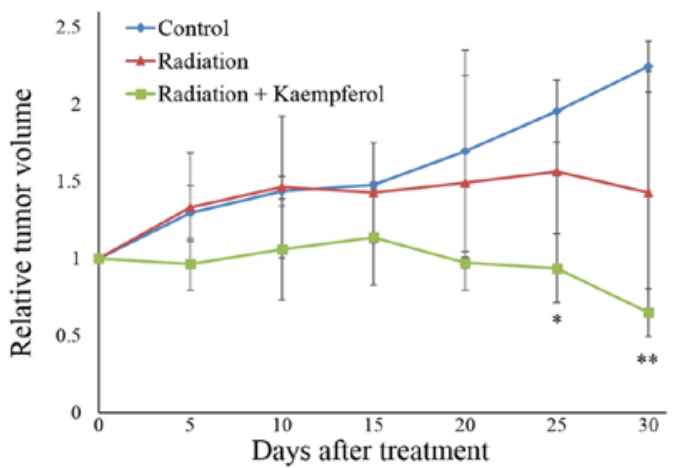

Figure 5. Effect of radiation only and radiation plus kaempferol on tumor volume in vivo. Treatment with radiation only and radiation plus $56 \mu \mathrm{M}$ kaempferol ( $4 \mathrm{~h}$ before radiation). The data shown are mean \pm SD for $n=3$. ${ }^{*} \mathrm{P}<0.05 ;{ }^{* * *} \mathrm{P}<0.01$ vs. control.
A
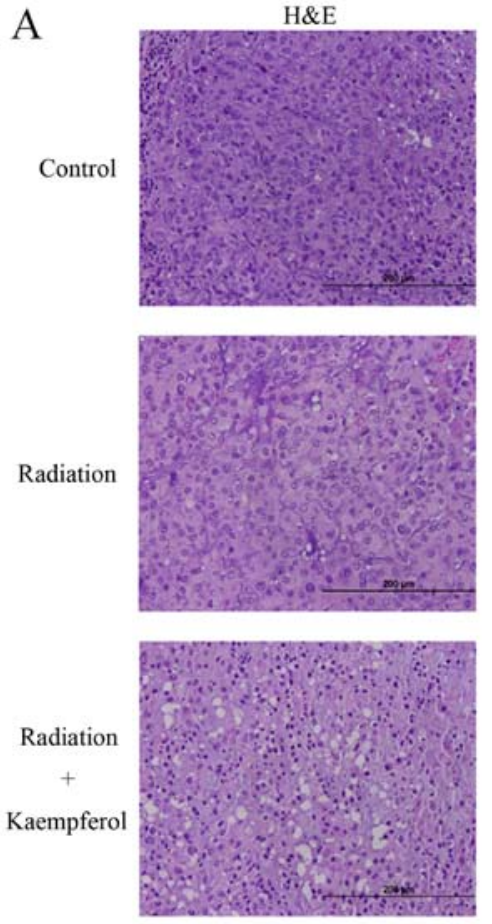

B

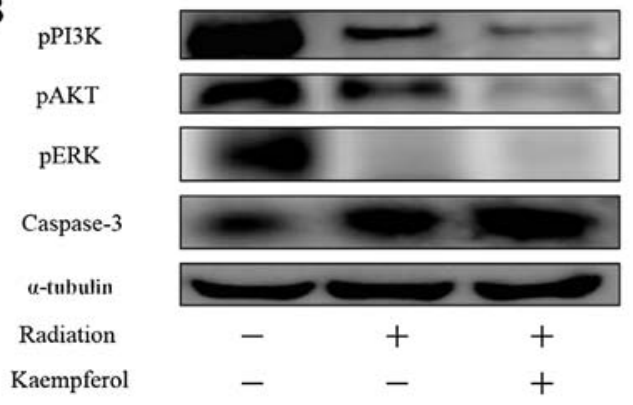

Figure 6. Effect of radiation only and radiation plus kaempferol on section staining and protein expression of tumor tissue. (A) $\mathrm{H} \& \mathrm{E}$ stain and IHC of caspase-3 (dark-brown). (B) pPI3K, pAKT, pERK and caspase-3 expression of extracted tumor tissues. $\mathrm{H} \& \mathrm{E}$, hematoxylin and eosin; IHC, immunohistochemistry.

dose enhancement factor (DEF) was 2, which was the ratio of the dose reducing the surviving fraction to 0.5 in the absence vs. presence of kaempferol. 
Kaempferol increases tumor cell killing of radiation in vivo. To determine the in vivo radiosensitivity of kaempferol, we used BALB/c nude mice with A-549 tumor xenografts. As shown in Fig. 5, treatment with radiation alone showed no significant difference in tumor growth compared to the control group (saline-treated). However, treatment with radiation plus kaempferol ( $4 \mathrm{~h}$ before radiation) resulted in a significant suppression of tumor growth 25 days after treatment. In addition, the tumor volume of the radiation plus kaempferol group decreased $35 \%$ of the starting volume 30 days after treatment.

Kaempferol enhances tumor cell apoptosis of radiation in vivo. To investigate histological differences between treated and control tumors, we performed tumor sections by H\&E staining and IHC of caspase-3 (Fig. 6A). Although control tumors exhibited a homogeneous distribution of viable cells, the sections of the radiation plus kaempferol-treated tumors showed apoptotic evidence of cell shrinkage, nuclear condensation and fragmentation via H\&E staining. IHC staining of caspase-3 was used to verify cell apoptosis (dark-brown cells). There was considerably more dark-brown staining of the radiation plus kaempferol group, compared with radiation only group.

Tumor tissue sections were extracted and processed for western blot analysis. As shown in Fig. 6B, treatment kaempferol plus radiation, not only inhibited the phosphorylation of PI3K, AKT and ERK, but also enhanced the expression of caspase-3, inducing tumor cell apoptosis.

\section{Discussion}

An important issue is to develop anticancer agents such as tumor-specific radiosensitizers that promote tumor radiosensitization to ultimately improve the therapeutic ratio and overcome tumor radioresistance. Kaempferol is a common flavonoid that is found in vegetables, fruits and tea $(25,26)$. Although kaempferol reportedly has anti-proliferative and cytotoxic effects on human lung cancer cells, to the best of our knowledge, no study has previously demonstrated that it can enhance radiosensitivity $(27,28)$. The present study provides evidence that kaempferol has radiosensitization potential for lung cancer in vitro and in vivo.

Kaempferol dose-dependently reduced the viability of A-549 cells, but had no significant effect on the normal HFL1 cell line. Such properties are relevant to the development of an anticancer drug that is not cytotoxic towards normal cells, unlike most currently used clinical drugs. Inhibition of the PI3K/AKT and ERK pathway may promote apoptosis proteins of Bcl-2 family members such as Bax, and then trigger caspase to induce cell apoptosis $(29,30)$. In the present stuyd, kaempferol suppressed the phosphorylation of AKT, PI3K and ERK, and then increased the expression of Bax, and subsequently stimulated caspase-7. The data demonstrate that kaempferol inhibited A-549 cells through activation of the mitochondria apoptosis pathway, which is consistent with the findings of a previous study (31).

Prior to investigating whether kaempferol was capable of enhancing the inhibitory effects of radiation on the growth of cancer cells, cell cycle regulation is important in mediating radiosensitivity. Cells have varying radiosensitivity in different phases of the cell cycle. The G2/M phase is most sensitive to radiation (32). In the current study, the cell population of $\mathrm{G} 2 / \mathrm{M}$ phase exhibited a time-dependent increase following treatment with kaempferol. Additionally, treatment with kaempferol prior to radiation exposure inhibited the growth of A-549 lung cancer cells more effectively. Kaempferol also inhibited the phosphorylation of PI3K and ERK sustainability after radiation exposure for $6 \mathrm{~h}$. We then verified the radiosentization effect of kaempferol using a clonogenic assay, which is also used to determine cell reproductive death after treatment with ionizing radiation (33). It was found that following pretreatment with kaempferol prior to radiation exposure, the surviving fraction decreased significantly. These data demonstrate that kaempferol has a radiosensitization potential for A-549 cells.

An animal model was used to determine the radiosensitization of kaempferol in vivo. Treatment with kaempferol prior to radiation $(4 \mathrm{~h})$ resulted in significant suppression of tumor growth and decreased tumor volume. Additionally, treatment with kaempferol prior to radiation enhanced the induction of cell apoptosis in tumor tissue by staining the histological sections. The protein level of the phosphorylation of AKT, PI3K and ERK from tumor tissue was also more inhibited following treatment with kaempferol plus radiation, and significantly activated caspase-3 to induce tumor apoptosis. The results demonstrate that kaempferol was able to increase in vivo tumor cell killing by radiation, indicating that kaempferol functions as a powerful radiosensitizer.

In conclusion, the results showed that, kaempferol increased tumor cell killing by radiation in vitro and in vivo through inhibition of the AKT/PI3K and ERK pathways and activation of the mitochondria apoptosis pathway. Thus, this study provided solid evidence that kaempferol is a safe and potential radiosensitizer for NSCLC.

\section{Acknowledgements}

The authors would like to thank the National Science Council of the Republic of China, Taiwan (contract no. NSC100-2628E-039-002-MY3) and the China Medical University (contract no. CMU100-S-36) for financially supporting this research.

\section{References}

1. Ferlay J, Shin HR, Bray F, Forman D, Mathers C and Parkin DM: Estimates of worldwide burden of cancer in 2008: GLOBOCAN 2008. Int J Cancer 127: 2893-2917, 2010.

2. Jemal A, Bray F, Center MM, Ferlay J, Ward E and Forman D: Global cancer statistics. CA Cancer J Clin 61: 69-90, 2011.

3. Herbst RS, Heymach JV and Lippman SM: Lung cancer. N Engl J Med 359: 1367-1380, 2008.

4. Pisters KM, Evans WK, Azzoli CG, Kris MG, Smith CA, Desch CE, Somerfield MR, Brouwers MC, Darling G, Ellis PM, et al; Cancer Care Ontario; American Society of Clinical Oncology: Cancer Care Ontario and American Society of Clinical Oncology adjuvant chemotherapy and adjuvant radiation therapy for stages I-IIIA resectable non small-cell lung cancer guideline. J Clin Oncol 25: 5506-5518, 2007.

5. Crinò L, Weder W, van Meerbeeck $J$ and Felip E; ESMO Guidelines Working Group: Early stage and locally advanced (non-metastatic) non-small-cell lung cancer: ESMO Clinical Practice Guidelines for diagnosis, treatment and follow-up. Ann Oncol 21 (Suppl 5): v103-v115, 2010.

6. Furuse K, Fukuoka M, Kawahara M, Nishikawa H, Takada Y, Kudoh S, Katagami N and Ariyoshi Y: Phase III study of concurrent versus sequential thoracic radiotherapy in combination with mitomycin, vindesine, and cisplatin in unresectable stage III non-small-cell lung cancer. J Clin Oncol 17: 2692-2699, 1999. 
7. Pfister DG, Johnson DH, Azzoli CG, Sause W, Smith TJ, Baker S Jr, Olak J, Stover D, Strawn JR, Turrisi AT, et al; American Society of Clinical Oncology: American Society of Clinical Oncology treatment of unresectable non-small-cell lung cancer guideline: Update 2003. J Clin Oncol 22: 330-353, 2004.

8. Eberhardt W, Pöttgen C and Stuschke M: Chemoradiation paradigm for the treatment of lung cancer. Nat Clin Pract Onco 3: 188-199, 2006.

9. Wardman P: Chemical radiosensitizers for use in radiotherapy. Clin Oncol (R Coll Radiol) 19: 397-417, 2007.

10. Kim IA, Bae SS, Fernandes A, Wu J, Muschel RJ, McKenna WG, Birnbaum MJ and Bernhard EJ: Selective inhibition of Ras, phosphoinositide 3 kinase, and Akt isoforms increases the radiosensitivity of human carcinoma cell lines. Cancer Res 65: 7902-7910, 2005.

11. Reardon DB, Contessa JN, Mikkelsen RB, Valerie K, Amir C, Dent $P$ and Schmidt-Ullrich RK: Dominant negative EGFRCD533 and inhibition of MAPK modify JNK1 activation and enhance radiation toxicity of human mammary carcinoma cells. Oncogene 18: 4756-4766, 1999.

12. Fokas E, Im JH, Hill S, Yameen S, Stratford M, Beech J, Hackl W, Maira SM, Bernhard EJ, McKenna WG, et al: Dual inhibition of the PI3K/mTOR pathway increases tumor radiosensitivity by normalizing tumor vasculature. Cancer Res 72: 239-248, 2012.

13. Wang T, Hu YC, Dong S, Fan M, Tamae D, Ozeki M, Gao Q Gius D and Li JJ: Co-activation of ERK, NF-kappaB, and GADD45beta in response to ionizing radiation. J Biol Chem 280 12593-12601, 2005

14. Kuo WT, Ho YJ, Kuo SM, Lin FH, Tsai FJ, Chen YS, Dong GC and Yao $\mathrm{CH}$ : Induction of the mitochondria apoptosis pathway by phytohemagglutinin erythroagglutinating in human lung cancer cells. Ann Surg Oncol 18: 848-856, 2011.

15. Miean KH and Mohamed S: Flavonoid (myricetin, quercetin, kaempferol, luteolin, and apigenin) content of edible tropical plants. J Agric Food Chem 49: 3106-3112, 2001.

16. Agarwal OP and Nagaratnam A: Radioprotective property of flavonoids in mice. Toxicon 19: 201-204, 1981.

17. Park JS, Rho HS, Kim DH and Chang IS: Enzymatic preparation of kaempferol from green tea seed and its antioxidant activity. J Agric Food Chem 54: 2951-2956, 2006.

18. Li W, Du B, Wang T, Wang S and Zhang J: Kaempferol induces apoptosis in human HCT116 colon cancer cells via the AtaxiaTelangiectasia Mutated-p53 pathway with the involvement of p53 upregulated modulator of apoptosis. Chem Biol Interact 177: 121-127, 2009.

19. Luo H, Rankin GO, Liu L, Daddysman MK, Jiang BH and Chen YC: Kaempferol inhibits angiogenesis and VEGF expression through both HIF dependent and independent pathways in human ovarian cancer cells. Nutr Cancer 61: 554-563, 2009.
20. DuPont MS, Day AJ, Bennett RN, Mellon FA and Kroon PA Absorption of kaempferol from endive, a source of kaempferol3-glucuronide, in humans. Eur J Clin Nutr 58: 947-954, 2004.

21. Nöthlings U, Murphy SP, Wilkens LR, Henderson BE and Kolonel LN: Flavonols and pancreatic cancer risk: The multiethnic cohort study. Am J Epidemiol 166: 924-931, 2007.

22. Leung HW, Lin CJ, Hour MJ, Yang WH, Wang MY and Lee HZ: Kaempferol induces apoptosis in human lung non-small carcinoma cells accompanied by an induction of antioxidant enzymes. Food Chem Toxicol 45: 2005-2013, 2007.

23. Jeong JC, Kim MS, Kim TH and Kim YK: Kaempferol induces cell death through ERK and Akt-dependent down-regulation of XIAP and survivin in human glioma cells. Neurochem Res 34: 991-1001, 2009.

24. Ackland ML, van de Waarsenburg S and Jones R: Synergistic antiproliferative action of the flavonols quercetin and kaempferol in cultured human cancer cell lines. In Vivo 19: 69-76, 2005.

25. Kowalski J, Samojedny A, Paul M, Pietsz G and Wilczok T: Effect of kaempferol on the production and gene expression of monocyte chemoattractant protein-1 in J774.2 macrophages. Pharmacol Rep 57: 107-112, 2005.

26. Sotibrán AN, Ordaz-Téllez MG and Rodríguez-Arnaiz R: Flavonoids and oxidative stress in Drosophila melanogaster. Mutat Res 726: 60-65, 2011

27. Moon SS, Rahman MA, Manir MM and Jamal Ahamed VS: Kaempferol glycosides and cardenolide glycosides, cytotoxic constituents from the seeds of Draba nemorosa (Brassicaceae). Arch Pharm Res 33: 1169-1173, 2010.

28. Xu X, Xie H, Hao J, Jiang Y and Wei X: Flavonoid glycosides from the seeds of Litchi chinensis. J Agric Food Chem 59: 1205-1209, 2011.

29. Cory S and Adams JM: The Bcl2 family: Regulators of the cellular life-or-death switch. Nat Rev Cancer 2: 647-656, 2002.

30. Fletcher JI, Meusburger S, Hawkins CJ, Riglar DT, Lee EF, Fairlie WD, Huang DC and Adams JM: Apoptosis is triggered when prosurvival Bcl-2 proteins cannot restrain Bax. Proc Natl Acad Sci USA 105: 18081-18087, 2008.

31. Nguyen TT, Tran E, Ong CK, Lee SK, Do PT, Huynh TT, Nguyen TH, Lee JJ, Tan Y, Ong CS, et al: Kaempferol-induced growth inhibition and apoptosis in A549 lung cancer cells is mediated by activation of MEK-MAPK. J Cell Physiol 197: 110-121, 2003.

32. Pawlik TM and Keyomarsi K: Role of cell cycle in mediating sensitivity to radiotherapy. Int J Radiat Oncol Biol Phys 59: 928-942, 2004.

33. Franken NA, Rodermond HM, Stap J, Haveman J and van Bree C: Clonogenic assay of cells in vitro. Nat Protoc 1: 2315-2319, 2006. 